\title{
Refractory Pancreatic Adenocarcinoma
}

National Cancer Institute

\section{Source}

National Cancer Institute. Refractory Pancreatic Adenocarcinoma. NCI Thesaurus. Code C162015.

Pancreatic adenocarcinoma that is resistant to treatment. 